\title{
ARITHMETIC OF DIVISION FIELDS
}

\author{
ARMAND BRUMER AND KENNETH KRAMER
}

(Communicated by Matthew A. Papanikolas)

\begin{abstract}
We study the arithmetic of division fields of semistable abelian varieties $A / \mathbb{Q}$. The Galois group of $\mathbb{Q}(A[2]) / \mathbb{Q}$ is analyzed when the conductor of $A$ is odd and squarefree. The irreducible semistable mod 2 representations of small conductor are determined under GRH. These results are used in our paper Paramodular abelian varieties of odd conductor.
\end{abstract}

\section{INTRODUCTION}

This paper contains results needed in [4] and of independent interest. We write $S$ for a set of primes, $N_{S}$ for their product and $\ell$ for a prime not in $S$. If $F / \mathbb{Q}$ is Galois, $\mathcal{I}_{v}(F / \mathbb{Q})$ denotes the inertia group at a place $v$ of $F$.

Definition $1.1([3])$. The Galois extension $F / \mathbb{Q}$ is $\left(\ell, N_{S}\right)$-controlled if

i) $F / \mathbb{Q}$ is unramified outside $S \cup\{\ell, \infty\}$;

ii) $\mathcal{I}_{v}(F / \mathbb{Q})=\left\langle\sigma_{v}\right\rangle$ is cyclic of order $\ell$ for all ramified $v$ not over $\ell$;

iii) $\mathcal{I}_{\lambda}(F / \mathbb{Q})^{u}=1$ for all $u>1 /(\ell-1)$ and $\lambda$ over $\ell$, using the upper ramification numbering as in $\$ 5$.

We denote by $V$ a finite dimensional vector space over the finite field $\mathbb{F}$ of characteristic $\ell$ with $q=|\mathbb{F}|$. Additional structure on $V$, such as a symplectic pairing or Galois action, is often imposed.

Definition 1.2. Let $V$ be an $\mathbb{F}\left[G_{\mathbb{Q}}\right]$-module and $F=\mathbb{Q}(V)$. The set $S$ of rational primes $p \neq \ell$ ramified in $F / \mathbb{Q}$ comprises the bad primes of $V$. Declare $V$ to be semistable if $F$ is $\left(\ell, N_{S}\right)$-controlled and $\left(\sigma_{v}-1\right)^{2}(V)=0$ for all $v$ lying over the primes of $S$.

Throughout, $A_{/ \mathbb{Q}}$ is a semistable abelian variety with good reduction at $\ell$ and End $_{\mathbb{Q}} A=\mathfrak{o}$ is the ring of integers in a totally real number field. If $\mathfrak{l}$ is a prime over $\ell$ in $\mathfrak{o}$ and $\mathfrak{o} / \mathfrak{l}=\mathbb{F}$, then $V=A[\mathfrak{l}]$ is semistable [9, 7]. The conductor of $A$ has the form $N_{A}=N^{d}$ with $d=[\mathfrak{o}: \mathbb{Z}]$. Since inertia over each bad prime $p$ is tame,

$$
\operatorname{ord}_{p}(N)=\operatorname{dim}_{\mathbb{F}} V / V^{\mathcal{I}}=\operatorname{dim}_{\mathbb{F}}\left(\sigma_{v}-1\right) V .
$$

In \$2 we use known results on symplectic representations generated by transvections to describe $\operatorname{Gal}(\mathbb{Q}(W) / \mathbb{Q})$ for constituents $W$ of $V$ with squarefree conductor, assuming $\mathfrak{l}$ lies over 2 .

Received by the editors March 26, 2011.

2010 Mathematics Subject Classification. Primary 11F80; Secondary 11S15, 11G10, 11Y40.

Key words and phrases. Semistable Galois representation, transvection, stem field discriminant, bounded ramification.

The research of the second author was partially supported by NSF grant DMS 0739346 . 
A stem field for a Galois extension $F / k$ is an intermediate field $K$ whose Galois closure over $k$ is $F$. If $G=\operatorname{Gal}(F / k)$ acts faithfully and transitively on a set $X$, the fixed field of the stabilizer $G_{x}$ of any $x$ in $X$ is a stem field. A formula for the discriminant $d_{K / k}$ is given in $\$$ and applied to semistable Galois modules. By relating number-theoretic properties of $K$ and $F$, certain computations may become feasible, since $K$ has a smaller degree and discriminant than $F$.

Suppose $E / \mathbb{Q}_{\ell}$ is a Galois extension of $\ell$-adic fields satisfying Definition 1.1(iii). In $\$ 5$, we find conditions on the ray class conductor of an abelian extension $L / E$ so that Definition 1.1(iii) also holds for the Galois closure of $L / \mathbb{Q}_{\ell}$. The maximal $(2, N)$-controlled extension for all odd $N \leq 79$ and for $N=97$ is determined in 46 , thanks to $\$$ and Odlyzko's GRH bounds. We also construct a $(2,127)$-controlled extension of degree 161280 with root discriminant just above the asymptotic Odlyzko bound, but finiteness of a maximal one is unknown to us.

A finite flat group scheme $\mathcal{V}$ over $\mathbb{Z}_{\ell}$ admits a filtration $0 \subseteq \mathcal{V}^{m} \subseteq \mathcal{V}^{0} \subseteq \mathcal{V}$ with connected component $\mathcal{V}^{0}$, étale quotient $\mathcal{V}^{e t}=\mathcal{V} / \mathcal{V}^{0}$, multiplicative subscheme $\mathcal{V}^{m}$ and biconnected subquotient $\mathcal{V}^{b}=\mathcal{V}^{0} / \mathcal{V}^{m}$. Let $\lambda$ be a place over $\ell$ in $F=\mathbb{Q}(V)$ and $\mathcal{D}_{\lambda}$ be its decomposition group. We denote the corresponding $\mathbb{F}\left[\mathcal{D}_{\lambda}\right]$-modules of $F_{\lambda}$-valued points by $V, V^{e t}, V^{m}$ and $V^{b}$, respectively.

Definition $1.4\left([4) . A_{/ \mathbb{Q}}\right.$ is o-paramodular if $\operatorname{dim} A=2 d$, with $d=[\mathfrak{o}: \mathbb{Z}]$.

Let $A$ be o-paramodular, with $\mathfrak{o} / \mathfrak{l} \simeq \mathbb{F}_{2}$. When $A[\mathfrak{l}]$ is irreducible, estimates for the discriminant of a stem field of $\mathbb{Q}(A[\mathfrak{l}])$ are obtained in $\$$. The reducible case leads to ray class fields whose conductors are controlled by the results of \$5. This information depends on the structure of $A[\mathfrak{l}]$ as a group scheme and is used in [4].

\section{Mod 2 Representations generated By transvections}

A transvection on $V$ is an automorphism of the form $\tau(x)=x+\psi(x) z$, with $\psi: V \rightarrow \mathbb{F}$ a non-zero linear form and $z \neq 0$ in ker $\psi$. Assume $V$ admits a nondegenerate alternating pairing [, ] $: V \times V \rightarrow \mathbb{F}$ preserved by $\tau$ and let $\operatorname{dim} V=2 n$. Then $\tau(x)=x+a[z, x] z$ for some $z \in V$ and $a \in \mathbb{F}^{\times}$. When $a$ is a square in $\mathbb{F}$, we may take $a=1$. For $x$ and $z$ in $V$, define $\tau_{[z]}$ by

$$
\tau_{[z]}(x)=x+[z, x] z .
$$

Assume that $\ell=2$ for the rest of this section, unless otherwise noted.

A quadratic form $\theta$ on the symplectic space $V$ is called a theta characteristic if $\theta(x+y)=\theta(x)+\theta(y)+[x, y]$ for all $x, y$ in $V$. Theta characteristics form a principal homogeneous space over $V$, with $(\theta+a)(x)=\theta(x)+[a, x]^{2}$ for $a$ in $V$. We identify $a$ with $[a,-]$ under the Galois isomorphism $V \simeq \operatorname{Hom}_{\mathbb{F}}(V, \mathbb{F})$. Elements $\sigma$ in $\operatorname{Sp}(V)$ act by $\sigma(\theta)(x)=\theta(\sigma(x))$. Then $\sigma(\theta+a)=\sigma(\theta)+\sigma(a)$ and

$$
\tau_{[z]}(\theta)=\theta+\sqrt{1+\theta(z)} z .
$$

Fix a symplectic basis $\left\{e_{1}, \ldots, e_{2 n}\right\}$ for $V$ with $\left[e_{i}, e_{j}\right]=1$ if $|i-j|=n$ and 0 otherwise. Let $\wp(x)=x^{2}-x$ be the Artin-Schreier function. Depending on whether or not the Arf invariant $\operatorname{Arf}(\theta)=\sum_{i} \theta\left(e_{i}\right) \theta\left(e_{i+n}\right)$ vanishes in $\mathbb{F} / \wp(\mathbb{F})$, we say $\theta$ is even or odd and write $O_{2 n}^{ \pm}$for the corresponding orthogonal group. Further, $\operatorname{Sp}(V)$ acts transitively on the sets $\Theta_{2 n}^{ \pm}$of even and odd characteristics and

$$
\left|\Theta_{2 n}^{ \pm}\right|=\frac{1}{2} q^{n}\left(q^{n} \pm 1\right)
$$


Denote the symmetric, alternating, dihedral and cyclic groups by $\mathcal{S}_{n}, \mathcal{A}_{n}, D_{n}$ and $C_{n}$ respectively.

Proposition 2.4 ([17]). If $\mathbb{F}=\mathbb{F}_{2}$ and $G \subsetneq \mathrm{SL}(\mathrm{V})$ is an irreducible subgroup generated by transvections, then $\operatorname{dim} V=2 n$ with $n \geq 2$ and $G$ is $O_{2 n}^{ \pm}\left(\mathbb{F}_{2}\right), \operatorname{Sp}_{2 n}\left(\mathbb{F}_{2}\right)$ or $\mathcal{S}_{m}$ with $2 n+1 \leq m \leq 2 n+2$. Also, $G$ has a trivial center and is self-normalizing in $\mathrm{SL}(\mathrm{V})$.

Proposition 2.5. Let $V$ be a symplectic space of dimension $2 n$. An irreducible subgroup $G$ of $\operatorname{Sp}(V)$ generated by transvections is one of the following, with $\mathbb{F}^{\prime} \subseteq \mathbb{F}$ :

i) dihedral, $D_{m}$ with $m$ dividing one of $|\mathbb{F}| \pm 1$ and $n=1$;

ii) orthogonal, $O_{2 n}^{ \pm}\left(\mathbb{F}^{\prime}\right)$ for $n \geq 2$;

iii) symplectic, $\mathrm{Sp}_{2 n}\left(\mathbb{F}^{\prime}\right)$;

iv) symmetric, $\mathcal{S}_{m}$ for $n \geq 2$ and $2 n+1 \leq m \leq 2 n+2$.

Moreover, $G$ has trivial center and is self-normalizing in $\operatorname{Sp}(V)$.

Proof. If $V$ is imprimitive, then $V$ is monomial [22], say $V=\operatorname{Ind}_{H_{1}}^{G}\left(V_{1}\right)$, with $V_{1}=\mathbb{F} e_{1}$ and $\left[G: H_{1}\right]=\operatorname{dim} V=2 n$. Arrange that $G=\bigcup g_{i} H_{1}$, with $g_{1}=1$ and $V_{i}=g_{i}\left(V_{1}\right)=\mathbb{F} e_{i}$, and let $\pi: G \rightarrow \mathcal{S}_{2 n}$ by $g V_{i}=V_{\pi(g) i}$. Since $\pi(G)$ is transitive and generated by transpositions, namely the images of the transvections, $\pi(G)=\mathcal{S}_{2 n}$. For $h$ in $H=\operatorname{ker} \pi$, we have $h e_{i}=\chi_{i}(h) e_{i}$, and so the pairing on $V$ satisfies $\left[e_{i}, e_{j}\right]=\left[h e_{i}, h e_{j}\right]=\chi_{i}(h) \chi_{j}(h)\left[e_{i}, e_{j}\right]$. Hence $\left[e_{i}, e_{j}\right]=0$ or $\chi_{i}(h) \chi_{j}(h)=$ 1. Because the pairing is perfect and $\pi(G)$ is doubly transitive, we must have $\left[e_{i}, e_{j}\right] \neq 0$ and $\chi_{i}(h) \chi_{j}(h)=1$ for all $i \neq j$. If $n \geq 2$, then $\chi_{i}(H)=1$ for all $i$, $H=1$ and $\pi$ is an isomorphism. The stabilizer $H_{1}$ of $V_{1}$ is isomorphic to $\mathcal{S}_{2 n-1}$, and so the character $\chi_{1}: H_{1} \rightarrow \mathbb{F}^{\times}$is trivial. Since $\sum g_{i}\left(e_{1}\right)$ is a non-trivial fixed point, $V$ is reducible. Now combine [11, Ch. II, §8.27] and [15, 16, to get our list.

If $g$ in $\operatorname{Sp}_{2 n}(\mathbb{F})$ normalizes $G$ and $\sigma$ is in $\operatorname{Gal}\left(\mathbb{F} / \mathbb{F}^{\prime}\right)$, then $g^{\sigma} g^{-1}$ centralizes $G$. Our representations are absolutely irreducible and the center of $\operatorname{Sp}_{2 n}(\mathbb{F})$ is trivial, so $g$ is in $\operatorname{Sp}_{2 n}\left(\mathbb{F}^{\prime}\right)$. To verify that the center is trivial and $G=\mathcal{S}_{m}$ is self-normalizing in $\mathrm{Sp}_{2 n}\left(\mathbb{F}_{2}\right)$ when $m \neq 6$, use the fact that all automorphisms are inner and absolute irreducibility. Note that $\mathcal{S}_{6} \simeq \mathrm{Sp}_{4}\left(\mathbb{F}_{2}\right)$. The dihedral case is easily checked. See [6] for the other cases.

Remark 2.6. As to (iv) above, note that $\mathcal{S}_{m}$ acts by permutation on

$$
Y=\left\{\left(a_{1}, \ldots, a_{m}\right) \in \mathbb{F}_{2}^{m} \mid a_{1}+\cdots+a_{m}=0\right\}
$$

with pairing $\left[\left(a_{i}\right),\left(b_{i}\right)\right]=\sum a_{i} b_{i}$. Let $V=Y /\langle(1, \ldots, 1)\rangle$ or $V=Y$ according as $m$ is even or odd. Then $V$ is irreducible and transpositions in $\mathcal{S}_{m}$ correspond to transvections on $V$. This action of $\mathcal{S}_{m}$ and that of Galois on $J[2]$ for a hyperelliptic Jacobian are compatible.

Lemma 2.7. Let $V$ be an irreducible $\mathbb{F}[G]$-module and let $P$ be the subgroup of $G$ generated by transvections. If $P$ is not trivial, then $V_{\mid P}$ is the direct sum of $r$ irreducible $\mathbb{F}[P]$-modules $W_{i}$ and $P=Q_{1} \cdots Q_{r}$ is a direct product, with $Q_{i}=\langle\sigma \in$ $P \mid \sigma_{\mid W_{i}}$ is a transvection and $\sigma_{\mid W_{j}}=1$ for all $\left.j \neq i\right\rangle$. If $V$ is symplectic, then the $W_{i}$ are symplectic and the sum is orthogonal.

Proof. Since $P$ is normal, Clifford's theorem applies. Let $W_{1}$ be an irreducible submodule of $V_{\mid P}, H=\left\{h \in G \mid h\left(W_{1}\right) \simeq W_{1}\right.$ as $P$-module $\}$ and $X=\sum_{h \in H} h\left(W_{1}\right)$. Then $V=\operatorname{ind}_{H}^{G}(X)$ and $X_{\mid P} \simeq e W_{1}$ is isotypic. If $G=\bigcup_{1}^{r} g_{i} H$ is a coset decomposition with $g_{1}=1$, then $V_{\mid P} \simeq \bigoplus_{1}^{r} e W_{i}$ with $W_{i}=g_{i}\left(W_{1}\right)$. For any transvection $\tau$, 
we have $1=\operatorname{dim}(\tau-1)(V)=e \sum_{1}^{r} \operatorname{dim}(\tau-1)\left(W_{i}\right)$. Thus $e=1$ and $\tau$ is in $Q_{i}$ for a unique index $i$. Moreover, $Q_{i}=g_{i} Q_{1} g_{i}^{-1}$ is normal in $P$ and $P=Q_{1} \cdots Q_{r}$ is a direct product.

Suppose $V$ is symplectic and $\tau$ is a transvection in $Q_{i}$. Then $(\tau-1) W_{i}=\langle z\rangle$ with $z$ in $W_{i} \cap W_{j}^{\perp}$ for all $j \neq i$, but not in $W_{i}^{\perp}$. Irreducibility of $W_{i}$ implies that $W_{i} \subseteq W_{j}^{\perp}$ and $W_{i} \cap W_{i}^{\perp}=0$. Hence $W_{i}$ is symplectic.

Proposition 2.8. Let $V$ be an irreducible symplectic $\mathbb{F}\left[G_{\mathbb{Q}}\right]$-module with squarefree conductor $N$ and let $F=\mathbb{Q}(V)$. Let $P$ be the subgroup of $G=\operatorname{Gal}(F / \mathbb{Q})$ generated by transvections. If $P=G$, then $G$ is as in Proposition 2.5.

Otherwise, $V=\operatorname{ind}_{P}^{G} W$ and $G \simeq Q \imath C_{2}$, where $Q$ is in the list in Proposition 2.5. Moreover, $F^{P}=\mathbb{Q}(i)$ and $N=\mathfrak{n} \overline{\mathfrak{n}}$ in $\mathbb{Z}[i]$, where $\mathfrak{n}$ generates the conductor ideal of $W$ as an $\mathbb{F}\left[G_{\mathbb{Q}(i)}\right]$-module.

Proof. Since $\operatorname{ord}_{p_{v}}(N)=1$, any generator $\sigma_{v}$ of $\mathcal{I}_{v}(F / \mathbb{Q})$ is a transvection. Proposition 6.2 shows that the fixed field $F^{P}=\mathbb{Q}(i)$. The restriction $V_{\mid P}$ is reducible by Lemma 2.5 and so $V$ is induced. Hence $H=P \simeq Q_{1} \times Q_{2}$ and $G \simeq Q_{1} \prec C_{2}$ is a wreath product, thanks to Lemma 2.7. The conductor formula for an induced module gives $N=\mathfrak{n} \overline{\mathfrak{n}}$, where $\mathfrak{n} \in \mathbb{Z}[i]$ is the odd part of the Artin conductor of $W$, since $\mathbb{Q}(i)$ is unramified at odd places.

Remark 2.9. In Proposition 2.8 if we take $\mathbb{F}=\mathbb{F}_{2}$ but do not assume $V$ symplectic, the conclusions hold, with "Proposition 2.5]' replaced by "Proposition 2.4]'.

Remark 2.10. The conjugacy class of any involution $\sigma$ in $\operatorname{Sp}(V)$ has invariants $t=\operatorname{rank}(\sigma-1)$ and $\delta$, with $\delta=0$ if $[v,(\sigma-1) v]=0$ for all $v$ in $V$ and $\delta=1$ otherwise. If $t=n$ and $\sigma$ is in $O_{2 n}^{-}(\mathbb{F})$, then $\delta=1$. If $t$ is odd, then $\delta=1$.

For the last result in this section, $\ell=3$.

Proposition 2.11. Let $V$ be an irreducible symplectic $\mathbb{F}_{3}\left[G_{\mathbb{Q}}\right]$-module with squarefree conductor $N$. Set $2 n=\operatorname{dim}_{\mathbb{F}} V, F=\mathbb{Q}(V)$ and $G=\operatorname{Gal}(F / \mathbb{Q})$. Then

i) $G \simeq \operatorname{GSp}_{2 n}\left(\mathbb{F}_{3}\right)$ or

ii) $n$ is even, $G \simeq \operatorname{Sp}_{n}\left(\mathbb{F}_{3}\right)$ < $C_{2}$ and $N=\mathfrak{n} \overline{\mathfrak{n}}$ in $\mathbb{Z}\left[\boldsymbol{\mu}_{3}\right]$.

Proof. An irreducible proper subgroup of $\mathrm{SL}_{2 n}\left(\mathbb{F}_{3}\right)$ generated by transvections is isomorphic to $\operatorname{Sp}_{2 n}\left(\mathbb{F}_{3}\right)$; cf. [16. The pairing on $V$ implies that $F$ contains $\boldsymbol{\mu}_{3}$. The subgroup $P$ of $G$ generated by all transvections fixes $K=\mathbb{Q}\left(\boldsymbol{\mu}_{3}\right)$ and $F^{P}$ is unramified outside $3 \infty$, so $F^{P}=K$ by Proposition 6.2. If $V_{\mid P}$ is irreducible, then (i) holds. If $V_{\mid P}$, is reducible, the arguments in the proofs of Lemma 2.7 and Proposition 2.8 give (ii), with $\mathfrak{n}$ a generator for the conductor ideal of the $\mathbb{F}\left[G_{\mathbb{Q}\left(\boldsymbol{\mu}_{3}\right)}\right]$-module $W$.

\section{DisCRIMinants OF STEM FIELDS}

Let $F / k$ be a Galois extension of number fields with group $G$. Let $\mathcal{D}$ be the decomposition group of a fixed prime $\pi_{F}$ of $F$ and $\mathcal{I}_{m}$ be the $m^{\text {th }}$ ramification group (see 95 ), with $\mathcal{I}=\mathcal{I}_{0}$ the inertia group. For intermediate fields $L$, set $\pi_{L}=\pi_{F} \cap L$. 
Theorem 3.1. Let $G$ act transitively on $X$. If $K$ is the fixed field of $G_{x}$ and $\mathcal{I}_{m} \backslash X$ is the set of $\mathcal{I}_{m}$-orbits of $X$, then

$$
\operatorname{ord}_{\pi_{k}}\left(d_{K / k}\right)=\sum_{m \geq 0} \frac{1}{\left[\mathcal{I}: \mathcal{I}_{m}\right]} \quad\left(|X|-\left|\mathcal{I}_{m} \backslash X\right|\right) .
$$

Proof. If $H=G_{x}$ and $I$ is any subgroup of $G$, then $H g I \leftrightarrow I g^{-1} x$ is a bijection between the set of double cosets $H \backslash G / I$ and the set of orbits $I \backslash X$. Thus,

$$
\sum_{H g I \in H \backslash G / I}\left[I:\left(I \cap H^{g}\right)\right]=[G: H],
$$

where $H^{g}=g^{-1} H g$. Suppose further that $J$ is a normal subgroup of $I$ so that $\left(I \cap H^{g}\right) J=I \cap H^{g} J$ is a subgroup of $I$. For each $g \in G$, we have

$$
H g I=\bigsqcup H g z_{i} J
$$

where $z_{i}$ runs over a set of representatives for the right cosets $I /\left(I \cap H^{g}\right) J$. The isomorphism $\left(I \cap H^{g}\right) /\left(J \cap H^{g}\right) \simeq\left(I \cap H^{g}\right) J / J$ implies that

$$
\begin{aligned}
\sum_{H g I \in H \backslash G / I} \frac{\left|J \cap H^{g}\right|}{\left|I \cap H^{g}\right|} & =\sum_{H g J \in H \backslash G / J} \frac{1}{\left[I:\left(I \cap H^{g}\right) J\right]} \frac{\left|J \cap H^{g}\right|}{\left|I \cap H^{g}\right|} \\
& =\sum_{H g J \in H \backslash G / J} \frac{1}{[I: J]}=\frac{|H \backslash G / J|}{[I: J]} .
\end{aligned}
$$

The ramification groups for $\pi_{F}$ inside $H$ are given by $\mathcal{I}_{m} \cap H$, and the different ideal $\mathfrak{D}_{F / k}$ satisfies $\operatorname{ord}_{\pi_{F}}\left(\mathfrak{D}_{F / k}\right)=\sum_{m=0}^{\infty}\left(\left|\mathcal{I}_{m}\right|-1\right)$. By transitivity of differents,

$$
\begin{aligned}
\operatorname{ord}_{\pi_{K}}\left(\mathfrak{D}_{K / k}\right) & =\frac{1}{|\mathcal{I} \cap H|} \operatorname{ord}_{\pi_{F}}\left(\mathfrak{D}_{K / k}\right) \\
& =\frac{1}{|\mathcal{I} \cap H|}\left(\operatorname{ord}_{\pi_{F}}\left(\mathfrak{D}_{F / k}\right)-\operatorname{ord}_{\pi_{F}}\left(\mathfrak{D}_{F / K}\right)\right) \\
& =\sum_{m \geq 0} \frac{\left|\mathcal{I}_{m}\right|-\left|\mathcal{I}_{m} \cap H\right|}{|\mathcal{I} \cap H|} .
\end{aligned}
$$

Each prime of $K$ over $\pi_{k}$ has the form $g\left(\pi_{F}\right) \cap K$, corresponding to a unique double coset $H g \mathcal{D}$ in $H \backslash G / \mathcal{D}$. Since the decomposition and inertia groups of $g\left(\pi_{F}\right)$ inside $G$ are $g \mathcal{D} g^{-1}$ and $g \mathcal{I} g^{-1}$, the ramification and residue degrees of $g\left(\pi_{F}\right) \cap K$ over $\pi_{k}$ are given by

$$
e(H g \mathcal{D})=\left[\mathcal{I}:\left(\mathcal{I} \cap H^{g}\right)\right] \text { and } f(H g \mathcal{D})=\left[\mathcal{D}:\left(\mathcal{D} \cap H^{g}\right) \mathcal{I}\right] .
$$

By conjugation, (3.5) implies that the exponent of $g\left(\pi_{F}\right) \cap K$ in $\mathfrak{D}_{K / k}$ is

$$
x(H g \mathcal{D})=\sum_{m \geq 0} \frac{\left|\mathcal{I}_{m}\right|-\left|\mathcal{I}_{m} \cap H^{g}\right|}{\left|\mathcal{I} \cap H^{g}\right|} .
$$

Moreover,

$$
\operatorname{ord}_{\pi_{k}}\left(d_{K / k}\right)=\sum_{H g \mathcal{D} \in H \backslash G / \mathcal{D}} x(H g \mathcal{D}) f(H g \mathcal{D}) .
$$


In view of (3.3) and (3.6), $H g \mathcal{D}$ is the disjoint union of $f(H g \mathcal{D})$ distinct elements of $H \backslash G / \mathcal{I}$. By (3.8) and (3.7), we now have

$$
\operatorname{ord}_{\pi_{k}}\left(d_{K / k}\right)=\sum_{H g \mathcal{I} \in H \backslash G / \mathcal{I}}=\sum_{m \geq 0} \sum_{H g \mathcal{I} \in H \backslash G / \mathcal{I}} \frac{\left|\mathcal{I}_{m}\right|-\left|\mathcal{I}_{m} \cap H^{g}\right|}{\left|\mathcal{I} \cap H^{g}\right|} .
$$

But (3.2) implies that

$$
\sum_{H g \mathcal{I} \in H \backslash G / \mathcal{I}} \frac{\left|\mathcal{I}_{m}\right|}{\left|\mathcal{I} \cap H^{g}\right|}=\sum_{H g \mathcal{I} \in H \backslash G / \mathcal{I}} \frac{\left[\mathcal{I}:\left(\mathcal{I} \cap H^{g}\right)\right]}{\left[\mathcal{I}: \mathcal{I}_{m}\right]}=\frac{[G: H]}{\left[\mathcal{I}: \mathcal{I}_{m}\right]}=\frac{[K: k]}{\left[\mathcal{I}: \mathcal{I}_{m}\right]},
$$

while (3.4) with $J=\mathcal{I}_{m}$ gives

$$
\sum_{H g \mathcal{I} \in H \backslash G / \mathcal{I}} \frac{\left|\mathcal{I}_{m} \cap H^{g}\right|}{\left|\mathcal{I} \cap H^{g}\right|}=\frac{\left|H \backslash G / \mathcal{I}_{m}\right|}{\left[\mathcal{I}: \mathcal{I}_{m}\right]} .
$$

Substituting the last two identities in the previous double sum proves our claim.

Corollary 3.9. If $\pi_{k}$ is tame in $F$, with ramification degree $|\mathcal{I}(F / k)|=\ell$ prime, then $\operatorname{ord}_{\pi_{k}}\left(d_{K / k}\right)=\left(1-\ell^{-1}\right)\left(|X|-\left|X^{\mathcal{I}}\right|\right)$.

Proof. Theorem 3.1 implies the claim, since $\mathcal{I}_{1}$ is trivial and there are $\left|X^{\mathcal{I}}\right|$ orbits of size 1 , while the others have size $\ell$.

We now apply these results to semistable $G_{\mathbb{Q}}$-modules $V$ of conductor $N$. We write $F=\mathbb{Q}(V)$ and $G=\operatorname{Gal}(F / \mathbb{Q})$.

Corollary 3.10. Let $t=\operatorname{ord}_{p}(N) \geq 1$ and $s=\operatorname{dim}_{\mathbb{F}} V$. If $G$ acts transitively on $X=V-\{0\}$ and $K=F^{G_{x}}$, then $\operatorname{ord}_{p}\left(d_{K / \mathbb{Q}}\right)=\left(1-\ell^{-1}\right)\left(q^{s}-q^{s-t}\right)$.

Proof. Our claim follows from Corollary 3.9, since $\operatorname{dim} V^{\mathcal{I}}=s-t$ by (1.3).

Now assume that $\ell=2$ and $V$ is symplectic of dimension $2 n$. Let $K$ be the fixed field of $G_{x}$, where $G$ acts transitively on $X$, as below:

i) $G \simeq \mathcal{S}_{m}=\operatorname{Sym}(X)$ and $V$ is the representation in Remark 2.6.

ii) $X=\Theta_{2 n}^{-}$or $X=\Theta_{2 n}^{-}-\left\{\theta_{0}\right\}$, with $\theta_{0}$ fixed by $G$.

Proposition 3.11. Let $\mathcal{I}_{v}=\langle\sigma\rangle \subseteq G$ be an inertia group at $v$ over $p \mid N$.

i) If $G \simeq \mathcal{S}_{m}$ and $\sigma$ is the product of $s$ disjoint transpositions, then $\operatorname{ord}_{p}\left(d_{K / \mathbb{Q}}\right)=$ $s$ and $\operatorname{ord}_{p}(N)=\min (s, n)$.

ii) If $G \simeq \operatorname{Sp}_{2 n}(\mathbb{F})$ or $O_{2 n}^{ \pm}(\mathbb{F})$, then $\operatorname{ord}_{p}\left(d_{K / \mathbb{Q}}\right)=\frac{1}{4} q^{n}\left(q^{n}-q^{n-t}-\delta\right)$, with $\delta$ as in Remark 2.10.

Proof. i) Since $\left|X^{\mathcal{I}_{v}}\right|=m-2 s$, we have $\operatorname{ord}_{p}\left(d_{K / \mathbb{Q}}\right)=s$ by Corollary 3.9 and, by (1.3) $\operatorname{ord}_{p}(N)=\operatorname{dim}_{\mathbb{F}}(\sigma-1)(V)=\min (s, n)$.

ii) We give a proof for $t=1$. Thus $\sigma$ is a transvection and we choose a symplectic basis for $V$ as in $\$ 2$, such that $\sigma=\tau_{\left[e_{n}\right]}$. For the even theta characteristic $\theta\left(x_{1}, \ldots, x_{2 n}\right)=\sum_{j=1}^{n} x_{j} x_{n+j}$, by (2.1) and (2.2), we have

$$
\sigma(\theta+a)=\theta+a+\left(1+\left[a, e_{n}\right]\right) e_{n} .
$$

Thus, $\sigma$ fixes $\theta+a$ if and only if $\left[a, e_{n}\right]=1$. Let $V^{\prime}=\left(\operatorname{span}\left\{e_{n}, e_{2 n}\right\}\right)^{\perp}$ and $\theta^{\prime}(y)=\sum_{j=1}^{n-1} y_{j} y_{n+j}$. Assume $\left[a, e_{n}\right]=1$ and write $a=y+a_{n} e_{n}+e_{2 n}$ with $y$ in $V^{\prime}$. In $\mathbb{F} / \wp(\mathbb{F})$, we have

$$
\operatorname{Arf}(\theta+a)=\operatorname{Arf}(\theta)+\theta(a)=a_{n}+\theta^{\prime}(y) .
$$


Hence $\theta+a$ is in $\Theta_{2 n}^{-}$precisely when one of the following conditions holds:
(a) $a_{n} \in \wp(\mathbb{F})$ and $\theta^{\prime}(y) \notin \wp(\mathbb{F}) \quad$ or
(b) $a_{n} \notin \wp(\mathbb{F})$ and $\theta^{\prime}(y) \in \wp(\mathbb{F})$.

If $n=1$, only (b) applies, yielding $\frac{1}{2} q$ choices of $a$. If $n \geq 2, y$ is in $\wp(\mathbb{F})$ exactly when $\theta^{\prime}+y$ is in $\Theta_{2 n-2}^{+}$. Hence there are $\frac{1}{2} q\left|\Theta_{2 n-2}^{-}\right|$choices of $a$ in case (a) and $\frac{1}{2} q\left|\Theta_{2 n-2}^{+}\right|$choices in case (b). But $\left|\Theta_{2 n-2}^{+}\right|+\left|\Theta_{2 n-2}^{-}\right|=\left|V^{\prime}\right|=q^{2 n-2}$, and so $\left|\left(\Theta_{2 n}^{-}\right)^{\mathcal{I}_{v}}\right|=\frac{1}{2} q^{2 n-1}$.

Definition 3.12. A semistable Galois module $V$ is ordinary at 2 if it is symplectic and $\mathfrak{a}^{2} V=0$, where $\mathfrak{a}$ is the augmentation ideal in $\mathbb{F}\left[\mathcal{I}_{\lambda}\right]$ for any $\lambda$ over 2 in $F$.

Let $V$ be the Galois module of a finite flat group scheme $\mathcal{V}$ over $\mathbb{Z}_{2}$. Then $\mathcal{I}_{\lambda}$ acts trivially on $V^{m}$ and $V^{e t}$. If the biconnected subquotient $\mathcal{V}^{b}$ is trivial, then $(\sigma-1)\left(\sigma^{\prime}-1\right)(V)=0$ for all $\sigma, \sigma^{\prime}$ in $\mathcal{I}_{\lambda}$, whence $V$ is ordinary. If $\mathcal{V}^{b} \neq 0$, then $\mathcal{I}_{\lambda}$ is not even a 2 -group.

We next treat the power of 2 in $d_{K / \mathbb{Q}}$ when $V$ is ordinary.

Lemma 3.13. We have $\mathfrak{a} V \subseteq Z \subseteq V^{\mathcal{I}_{\lambda}}$ for some maximal isotropic subspace $Z$ of $V$. If $H=G_{\theta}$ stabilizes an odd theta characteristic $\theta$, then $\left|\mathcal{I}_{\lambda} /\left(\mathcal{I}_{\lambda} \cap H\right)\right| \leq \frac{1}{2} q^{n}$.

Proof. Set $\mathcal{I}=\mathcal{I}_{\lambda}$. Since $\mathfrak{a}^{2} V=0$ and $\mathcal{I} \subseteq \operatorname{Sp}(V)$, we find $\mathfrak{a} V \subseteq V^{\mathcal{I}}=(\mathfrak{a} V)^{\perp}$. Thus, $\mathfrak{a} V$ is contained in a maximal isotropic space $Z$ and, by duality, $Z \subseteq V^{\mathcal{I}}$.

If $\Gamma$ is the subgroup of $\operatorname{Sp}_{2 n}(\mathbb{F})$ fixing both $Z$ and $V / Z$ pointwise, then we have $(g-1)\left(g^{\prime}-1\right)(V)=0$ for all $g, g^{\prime}$ in $\Gamma$. Hence $\psi(g)=(g-1) \theta$ defines a homomorphism $\Gamma \rightarrow V$. In the notation of (2.1), $\Gamma$ is generated by the transvections $\tau_{[z]}$ with $z$ in $Z$. Since we may identify $\left(\tau_{[z]}-1\right) \theta$ with $\sqrt{1+\theta(z)} z$, the homomorphism $\psi$ takes values in $Z$. We next verify the exactness of the sequence

$$
0 \rightarrow \Gamma \cap H \rightarrow \Gamma \stackrel{\psi}{\rightarrow} Z \stackrel{\theta}{\rightarrow} \mathbb{F} / \wp \mathbb{F} \rightarrow 0 .
$$

Since $Z$ is isotropic, $\theta$ is linear on $Z$ and $\theta$ is surjective because it is odd. Clearly $\theta\left(\psi\left(\tau_{[z]}\right)\right)$ is in $\wp \mathbb{F}$. Conversely, if $\theta(z)=a^{2}+a$ and $y=(1 / \sqrt{a}) z$, then $\psi\left(\tau_{[y]}\right)=z$. This proves exactness around $Z$, and the rest is clear.

Finally, $\mathcal{I} \subseteq \Gamma$, and therefore $|\mathcal{I} /(\mathcal{I} \cap H)| \leq|\Gamma /(\Gamma \cap H)|=\frac{1}{2} q^{n}$.

Proposition 3.15. If $V$ is ordinary at 2 and $G$ is transitive on $\Theta_{2 n}^{-}$or $\Theta_{2 n}^{-}-\left\{\theta_{0}\right\}$, then $\operatorname{ord}_{2}\left(d_{K / \mathbb{Q}}\right) \leq\left(q^{n}-2\right)\left(q^{n}-1-\epsilon\right)$, where $\epsilon=0$ or 1 , respectively.

Proof. Since $\mathcal{I}$ is a 2-group, $\mathcal{I}_{0}=\mathcal{I}_{1}$. The definition of the upper numbering (see 95) and the bound on wild ramification (Definition 1.1(iii)) imply that $\mathcal{I}_{2}=1$. By Theorem 3.1, $\operatorname{ord}_{2}\left(d_{K / \mathbb{Q}}\right)=2(|X|-|\mathcal{I} \backslash X|)$.

By Lemma 3.13, each $\mathcal{I}$-orbit of $X$ has at most $\frac{1}{2} q^{n}$ elements and there are at least $2\left|\Theta_{2 n}^{-}\right| / q^{n}=q^{n}-1$ orbits when $\epsilon=0$, proving the claim.

If $\epsilon=1, \mathcal{I}$ fixes $\theta_{0}$. The theta characteristic $\theta_{0}+z$ is odd exactly if $\theta_{0}(z)$ is in $\wp \mathbb{F}$. By (3.14), there are $\frac{1}{2} q^{n}$ such $z \in Z$, giving at least $\frac{1}{2} q^{n}-1$ orbits of size 1 for $\mathcal{I}$ acting on $X$. The number of orbits not accounted for is at least

$$
\frac{|X|-\left(\frac{1}{2} q^{n}-1\right)}{\frac{1}{2} q^{n}}=q^{n}-2,
$$

and so $|\mathcal{I} \backslash X| \geq \frac{1}{2} q^{n}-1+\left(q^{n}-2\right)=\frac{3}{2} q^{n}-3$. Hence our claim.

Proposition 3.16. If $V$ is ordinary and $G$ is a transitive subgroup of $\mathcal{S}_{m}$, then $\operatorname{ord}_{2}\left(d_{K / \mathbb{Q}}\right) \leq 2\lfloor m / 2\rfloor$, unless $m=4$ or 8 , when $\operatorname{ord}_{2}\left(d_{K / \mathbb{Q}}\right) \leq 3 m / 2$. 
Proof. We find lower bounds for the number of $\mathcal{I}$-orbits and apply Theorem 3.1 . Since there is at least one orbit, our claims hold for $m \leq 4$. Assume $m \geq 5$ and refer to the explicit representation (2.6). Let $y_{i, j} \in Y$ denote the vector with non-zero entries only in coordinates $i$ and $j$. Write $\bar{y} \in V$ for the coset of $y \in Y$ when $m$ is even and $\bar{y}=y$ otherwise.

Suppose distinct letters $i, j$ lie in the same $\mathcal{I}$-orbit. If we can find a permutation $\sigma$ in $\mathcal{I}$ such that $\sigma(i)=j$ and $\sigma(k)=k$, then $\bar{y}_{i, j}=(\sigma-1)\left(\bar{y}_{i, k}\right) \in \mathfrak{a} V$ is fixed by $\mathcal{I}$. It follows that $\tau\left(y_{i, j}\right)=y_{i, j}$ for all $\tau$ in $\mathcal{I}$, and so $\{i, j\}$ is an $\mathcal{I}$-orbit.

A larger orbit can exist only if $m=2 n+2$ is even and $\mathcal{I}$ contains a product of $n+1$ disjoint transpositions, say

$$
\sigma=(1, n+2)(2, n+3) \cdots(n+1,2 n+2) .
$$

Treat subscripts modulo $2 n+2$, fix $k$ and consider $j \notin\{k, k+n+1\}$. Then

$$
\bar{x}_{j}:=\bar{y}_{j, j+n+1}-\bar{y}_{k, k+n+1}=(\sigma-1)\left(\bar{y}_{j, k}\right) \in \mathfrak{a} V
$$

is fixed by $\mathcal{I}$. If $m \neq 8, \bar{x}_{j}$ has a unique representative $x_{j} \in Y$ with exactly 4 non-zero entries, and so $\tau\left(x_{j}\right)=x_{j}$ for all $\tau$ in $\mathcal{I}$. Since

$$
\tau(k) \in \bigcap_{j \notin\{k, k+n+1\}}\{j, j+n+1, k, k+n+1\}=\{k, k+n+1\},
$$

$\{k, k+n+1\}$ is an $\mathcal{I}$-orbit and every $\mathcal{I}$-orbit has 2 elements. If $m=8$, the $\mathcal{I}$-orbits have size at most 4 , giving the weaker bound.

\section{Stem field discriminant for $\mathbb{Q}(A[\mathfrak{l}])$ in a special Case}

In this section, $A_{/ \mathbb{Q}}$ is o-paramodular with good reduction at $2, \mathfrak{l}$ is a prime of $\mathfrak{o}$ with residue field $\mathbb{F}_{2}$ and $V=A[\mathfrak{l}]$ is irreducible. Thus $V$ admits a symplectic pairing [4, §3]. Let $F=\mathbb{Q}(V)$ and $G=\operatorname{Gal}(F / \mathbb{Q})$. The elements of $V$ correspond to differences $\theta_{i}-\theta_{j}$ of the 6 odd theta characteristics, and we view $G$ as a subgroup of $\mathcal{S}_{6}$, via its action on $\Theta^{-}$. Irreducibility of $V$ implies that $G$ has an orbit $\Sigma \subseteq \Theta^{-}$ of size 5 or 6 . If $H=G_{\theta}$ stabilizes $\theta$ in $\Sigma$, then $K=F^{H}$ is a stem field for $F$, with $[K: \mathbb{Q}]=|\Sigma|$.

The following local building blocks are used in the next result. Let $X$ be the irreducible $G_{\mathbb{Q}_{2}}$-module such that $\operatorname{dim}_{\mathbb{F}_{2}} X=2$ and $\tilde{E}=\mathbb{Q}_{2}(X)=\mathbb{Q}_{2}\left(\boldsymbol{\mu}_{3}, \sqrt[3]{2}\right)$. The exhaustive list [13] of 2-adic fields of low degree, or class field theory, shows that there is a unique quartic extension $\tilde{M} / \mathbb{Q}_{2}$ whose Galois closure $\tilde{L}$ has nontrivial tame ramification, necessarily of degree 3 . Then $\tilde{M} / \mathbb{Q}_{2}$ is totally ramified, $\operatorname{ord}_{2}\left(d_{\tilde{M} / \mathbb{Q}_{2}}\right)=4, \tilde{L}$ contains $\tilde{E}$ and $\operatorname{Gal}\left(\tilde{L} / \mathbb{Q}_{2}\right) \simeq \mathcal{S}_{4}$, with inertia subgroup $\mathcal{A}_{4}$.

Proposition 4.1. $\operatorname{ord}_{2}\left(d_{K / \mathbb{Q}}\right) \leq 4$ (resp. 6) if $[K: \mathbb{Q}]=5$ (resp. 6).

Proof. If $V$ is ordinary at 2, the result follows from Proposition 3.15 or 3.16. Hence we suppose $F$ has non-trivial tame ramification over 2. Among primes over 2 in $K$, choose $\lambda$ with maximal ramification degree $e_{\lambda}(K)$ and consider all possibilities:

i) $e_{\lambda}(K)=5$. Then $(2) \mathcal{O}_{K}=\lambda^{5}$ or $\lambda^{5} \lambda^{\prime}$, depending on whether $K$ is quintic or sextic, and $\operatorname{ord}_{2}\left(d_{K / \mathbb{Q}}\right)=4$ by tame theory.

ii) $e_{\lambda}(K)=3$. If $K$ is quintic, the worst case occurs when $(2) \mathcal{O}_{K}=\lambda^{3}\left(\lambda^{\prime}\right)^{2}$, and then we have $\operatorname{ord}_{2}\left(d_{K / \mathbb{Q}}\right)=\operatorname{ord}_{2}\left(d_{K_{\lambda} / \mathbb{Q}_{2}}\right)+\operatorname{ord}_{2}\left(d_{K_{\lambda^{\prime}} / \mathbb{Q}_{2}}\right)=2+2=4$. Suppose $K$ is sextic. If $(2) \mathcal{O}_{K}=\left(\lambda \lambda^{\prime}\right)^{3}$ or $\lambda^{3}$ with residue degree $f_{\lambda}(K)=2$, we have 
$\operatorname{ord}_{2}\left(d_{K / \mathbb{Q}}\right)=4$. In the remaining cases, at most one more prime $\lambda^{\prime}$ over 2 ramifies in $K$, with $e_{\lambda^{\prime}}(K)=2$, and we conclude as for quintics.

iii) $e_{\lambda}(K)=4$. Then the completion $K_{\lambda}=\tilde{M}$. If $[K: \mathbb{Q}]=5$, the other prime over 2 in $K$ is unramified, but if $[K: \mathbb{Q}]=6$, there may at worst be some $\lambda^{\prime}$ with $e_{\lambda^{\prime}}(K)=2$. Hence

$$
\operatorname{ord}_{2}\left(d_{K / \mathbb{Q}}\right) \leq \begin{cases}4 & \text { if }[K: \mathbb{Q}]=5 \\ 4+2=6 & \text { if }[K: \mathbb{Q}]=6\end{cases}
$$

iv) $e_{K}(\lambda)=6$, so $[K: \mathbb{Q}]=6,(2) \mathcal{O}_{K}=\lambda^{6}$ and the inertia group $\mathcal{I}$ of $\lambda$ acts transitively on $\Theta^{-}$. Since a non-zero fixed point for the action of $\mathcal{I}$ on $V$ corresponds to a pair of theta characteristics preserved by $\mathcal{I}$, contradicting transitivity, there are none. The tame ramification group $\mathcal{I} / \mathcal{I}_{1}$ is a cyclic subgroup of $\mathcal{S}_{6}$ whose order is odd and a multiple of 3 . Hence $\left|\mathcal{I} / \mathcal{I}_{1}\right|=3$.

Because $\mathcal{I}_{1}$ is a non-trivial 2-group, normal in its decomposition group $\mathcal{D}$, the fixed space $W=V^{\mathcal{I}_{1}}$ is a non-zero $\mathcal{D}$-module, properly contained in $V$. Viewed as an $\mathcal{I} / \mathcal{I}_{1}$-module, $W$ is semisimple. But $\mathcal{I} / \mathcal{I}_{1}$ has no non-zero fixed points on $W$, as they would be fixed points of $\mathcal{I}$, so $\operatorname{dim} W=2$ and $W \simeq X$.

Viewed as a finite flat group scheme over $\mathbb{Z}_{2}, \mathcal{V}=A[\mathfrak{l}]$ is Cartier self-dual. The multiplicative component $\mathcal{V}^{m}$ cannot have order 4, since $\mathcal{I}$ is not a 2-group, nor can it have order 2 , since $\mathcal{I}$ has no non-trivial fixed points. Hence $\mathcal{V}^{m}=0$ and $\mathcal{V}$ is fully biconnected. There is a subgroup scheme $\mathcal{W}$ of $\mathcal{V}$ with $\mathcal{D}$-module $W$, and $\mathcal{V} / \mathcal{W}$ is biconnected, so its $\mathcal{D}$-module also is isomorphic to $X$.

Schoof [20, Prop, 6.4] showed that if $V$ is an extension of $X$ by $X$ as a $\mathcal{D}$-module, then $\mathbb{Q}_{2}(V)$ is contained in the maximal elementary 2-extension $\tilde{L}_{1}$ of $\tilde{E}$ with ray class conductor exponent 2 . One checks that $\tilde{L}_{1}$ is an unramified central extension of degree 2 over $\tilde{L}$ and the root discriminant of $\tilde{L}_{1} / \mathbb{Q}_{2}$ is $7 / 6$. Since $\operatorname{ord}_{2}\left(d_{K / \mathbb{Q}}\right)$ is even, we have $\operatorname{ord}_{2}\left(d_{K / \mathbb{Q}}\right) \leq 6$, as claimed.

\section{Preserving the Fontaine Bound}

Let $K^{\prime} / K$ be a Galois extension of $\ell$-adic fields with Galois group $G$. Denote the ring of integers of $K^{\prime}$ by $\mathcal{O}^{\prime}$ and a prime element by $\lambda^{\prime}$. Set

$$
G_{n}=\left\{\sigma \in G \mid \operatorname{ord}_{\lambda^{\prime}}(\sigma(x)-x) \geq n+1 \text { for all } x \in \mathcal{O}^{\prime}\right\},
$$

so $G_{0}$ is the inertia group and $t_{K^{\prime} / K}=\left[G_{0}: G_{1}\right]$ is the degree of tame ramification. If $\lfloor x\rfloor=m$, the Herbrand function is given by

$$
\varphi_{K^{\prime} / K}(x)=\frac{1}{\left|G_{0}\right|}\left(\left|G_{1}\right|+\cdots+\left|G_{m}\right|+(x-m)\left|G_{m+1}\right|\right)
$$

and is continuous and increasing. In the upper numbering used by Serre [21, IV], $G^{m}=G_{n}$, with $m=\varphi_{K^{\prime} / K}(n)$. In the numbering of [7] or [13], this group is denoted by $G^{(m+1)}$. Let $\psi_{K^{\prime} / K}$ be the inverse of $\varphi_{K^{\prime} / K}$.

Notation 5.2. Let $c=c_{K^{\prime} / K}$ be the maximal integer such that $G_{c} \neq 1$. We omit the lower field if $K=\mathbb{Q}_{\ell}$. Let $m_{K^{\prime}}=\psi_{K^{\prime} / \mathbb{Q}_{\ell}}\left(\frac{1}{\ell-1}\right)$.

Wild ramification in $K^{\prime} / K$ is equivalent to $c_{K^{\prime} / K} \geq 1$. If $G_{1}$ is not abelian, then $c_{K^{\prime} / K} \geq 2$, since successive quotients in the ramification filtration are elementary abelian $\ell$-groups. By (5.1), $m_{K^{\prime}}$ is an integer when $(\ell-1)$ divides $t_{K^{\prime}} / \mathbb{Q}_{\ell}$. 
Lemma 5.3. Let $E \supset F$, both Galois over $K, G=\operatorname{Gal}(E / K)$ and $H=\operatorname{Gal}(E / F)$. Then $1 \rightarrow H^{\psi_{F / K}(x)} \rightarrow G^{x} \stackrel{\text { res }}{\longrightarrow} \operatorname{Gal}(F / K)^{x} \rightarrow 1$ is exact. In addition,

$$
m_{E} \geq t_{E / F} m_{F} \quad \text { and } \quad c_{E / K} \geq t_{E / F} c_{F / K} .
$$

Proof. By compatibility with quotients, res is surjective and its kernel is

$$
G^{x} \cap H=G_{\psi_{E / K}(x)} \cap H=H_{\psi_{E / K}(x)}=H^{\varphi_{E / F} \psi_{E / K}(x)}=H^{\psi_{F / K}(x)},
$$

since $\psi_{E / K}=\psi_{E / F} \psi_{F / K}$. Thus the sequence is exact.

Equation (5.1) implies that $t_{E / F} \varphi_{E / F}(z) \leq z$, so $\psi_{E / F}(z) \geq t_{E / F} z$. If $x=$ $\varphi_{F / K}\left(c_{F / K}\right)$, then $G_{\psi_{E / K}(x)}=G^{x} \neq 1$ by surjectivity of res. Hence

$$
c_{E / K} \geq \psi_{E / K}(x)=\psi_{E / F} \psi_{F / K}(x)=\psi_{E / F}\left(c_{F / K}\right) \geq t_{E / F} c_{F / K},
$$

and similarly for $m_{E} \geq t_{E / F} m_{F}$.

Definition 5.5. Let $F$ be the Galois closure of $K / \mathbb{Q}_{\ell}$. We say $K$ is Fontaine if $\operatorname{Gal}\left(F / \mathbb{Q}_{\ell}\right)^{u}=1$ for all $u>\frac{1}{\ell-1}$ or, equivalently, $c_{F} \leq m_{F}$.

Lemma 5.6. Let $E \supset F$, both Galois over $\mathbb{Q}_{\ell}, G=\operatorname{Gal}\left(E / \mathbb{Q}_{\ell}\right)$ and $H=\operatorname{Gal}(E / F)$. Then:

i) If $t_{F / \mathbb{Q}_{\ell}}=\ell-1$, then $m_{E} \geq t_{E / F}$, with equality when $G_{0}$ is abelian.

ii) Let $F$ be Fontaine, with non-trivial wild ramification. Then $1 \leq c_{F} \leq m_{F}$. Assume further that $t_{F / \mathbb{Q}_{\ell}}=\ell-1$. Then $c_{F}=m_{F}=1$ and, if $E$ is Fontaine, then $c_{E}=m_{E}$.

Proof. i) Since $\varphi_{F / \mathbb{Q}_{\ell}}(1)=\frac{1}{\ell-1}$, we have $m_{F}=1$, so $m_{E} \geq t_{E / F}$ by (5.4). If $G_{0}$ is abelian and $t_{E / \mathbb{Q}_{\ell}}$ does not divide $j$, then $G_{j}=G_{j+1}$ by [21, IV, §2]. Thus the definition gives $\varphi_{E / \mathbb{Q}_{\ell}}\left(t_{E / F}\right)=\frac{1}{\ell-1}$, whence $m_{E}=t_{E / F}$.

ii) By Definition [5.5. $\varphi_{F / \mathbb{Q}_{\ell}}\left(c_{F}\right) \leq \frac{1}{\ell-1}=\varphi_{F / \mathbb{Q}_{\ell}}\left(m_{F}\right)$. Hence $c_{F} \leq m_{F}$. If $t_{F / \mathbb{Q}_{\ell}}=\ell-1$, then $m_{F}=1$, so $c_{F}=1$. Surjectivity of res in Lemma 5.3 implies that $G^{\frac{1}{\ell-1}} \neq 1$. If $E$ is Fontaine, it follows that $c_{E}=\psi_{E / \mathbb{Q}_{\ell}}\left(\frac{1}{\ell-1}\right)=m_{E}$.

Example 5.7. By class field theory or the table of quartics 13 , there is a unique Fontaine $\mathcal{S}_{4}$-extension $F / \mathbb{Q}_{2}$. The ramification subgroups of $\bar{G}=\operatorname{Gal}\left(F / \mathbb{Q}_{2}\right)$ are $\bar{G}_{0} \simeq \mathcal{A}_{4}, \bar{G}_{1} \simeq C_{2}^{2}$ and $\bar{G}_{2}=1$, so $c_{F}=1, \varphi_{F / \mathbb{Q}_{2}}(x)=(4+(x-1)) / 12$ if $x \geq 1$ and $m_{F}=9$. Moreover, $E=F(i)$ remains Fontaine, with $G=\operatorname{Gal}\left(E / \mathbb{Q}_{2}\right) \simeq \mathcal{S}_{4} \times C_{2}$. Lemma 5.6(ii) may be used to show that $\left|G_{0}\right|=24,\left|G_{1}\right|=8,\left|G_{2}\right|=\cdots=\left|G_{9}\right|=2$, $\left|G_{10}\right|=1$ and $c_{E}=m_{E}=9$. Alternatively, $E$ has two stem fields of degree 6 , and this determines $E$ uniquely in [13].

Lemma 5.8. Let $M / F$ be abelian, with $F / \mathbb{Q}_{\ell}$ Galois. Then $M$ is Fontaine if and only if $F$ is Fontaine and the ray class conductor exponent $\mathfrak{f}(M / F) \leq\left\lfloor m_{F}\right\rfloor+1$.

Proof. If $E$ is the Galois closure of $M / \mathbb{Q}_{\ell}$, then $E / F$ is abelian and we have $\mathfrak{f}(E / F)=\mathfrak{f}(M / F)=\varphi_{E / F}\left(c_{E / F}\right)+1 ;$ cf. [21, XV, 22$]$. The exact sequence of Lemma 5.3 with $K=\mathbb{Q}_{\ell}$ implies our claim.

Remark 5.9. Let $E$ be a number field with root discriminant $\varrho_{E}$. Write $\tilde{E}$ for the completion of $E$ at a prime $\lambda \mid \ell$ and $e_{\tilde{E}}$ for the absolute ramification degree. Suppose $E$ contains $F$, both Galois over $\mathbb{Q}$, with $\tilde{E}$ Fontaine. Then

$$
\operatorname{ord}_{\ell}\left(\varrho_{E}\right) \leq 1+\frac{1}{\ell-1}-\frac{t_{\tilde{E} / \tilde{F}} c_{\tilde{F}}+1}{e_{\tilde{E}}} .
$$


Indeed, if $\mathfrak{D}_{\tilde{E} / \mathbb{Q}_{\ell}}$ is the different, then [21, IV, Prop. 4] and [7, Prop. 1.3] give

$$
\operatorname{ord}_{\ell}\left(\varrho_{E}\right)=\frac{1}{e_{\tilde{E}}} \operatorname{ord}_{\lambda}\left(\mathfrak{D}_{\tilde{E} / \mathbb{Q}_{\ell}}\right)=1+\varphi_{\tilde{E} / \mathbb{Q}_{\ell}}\left(c_{\tilde{E}}\right)-\frac{c_{\tilde{E}}+1}{e_{\tilde{E}}} .
$$

We conclude by Definition 5.5] and equation (5.4).

Because the upper numbering is compatible with quotients, the composition of Fontaine fields is Fontaine and there is a maximal field $L$, such that $\operatorname{Gal}\left(F / \mathbb{Q}_{\ell}\right)^{u}=1$ for all Galois subfields $F$ finite over $\mathbb{Q}_{\ell}$ and all $u>\frac{1}{\ell-1}$. Since $F=\mathbb{Q}_{\ell}\left(\boldsymbol{\mu}_{\ell},(1-\ell)^{\frac{1}{\ell}}\right)$ is contained in $L$, Lemma 5.6(ii) implies a gap in the upper numbering:

$$
\operatorname{Gal}\left(L / \mathbb{Q}_{\ell}\right)^{\frac{1}{\ell-1}} \neq \operatorname{Gal}\left(L / \mathbb{Q}_{\ell}\right)^{\frac{1}{\ell-1}+\epsilon} \text { for } \epsilon>0 .
$$

Hajir and Maier [10] study number field extensions $K^{\prime} / K$ of bounded depth, i.e., with vanishing ramification groups $\mathcal{D}_{\mathfrak{p}}\left(K^{\prime} / K\right)^{x}$ for all $x \geq \nu_{\mathfrak{p}}$. When there is deep wild ramification, the concept of Galois slope content introduced by Jones and Roberts [13] and used in [12, §1.4] leads to variants of (5.4) and Remark 5.9, not required for our applications, thanks to Definition 1.1(iii).

\section{Using ODLYZKo}

We study some maximal $(\ell, N)$-controlled extensions $L / \mathbb{Q}$ by means of Odlyzko's bounds [18, 19, 5]. If the $\mathbb{F}\left[G_{\mathbb{Q}}\right]$-module $V$ is semistable and bad only at $S$, then $\mathbb{Q}(V)$ is $\left(\ell, N_{S}\right)$-controlled. The converse holds for $\ell=2$ but not for $\ell$ odd; e.g., if $\operatorname{dim} V=2$, then $\operatorname{Sym}^{2} V$ rarely is semistable.

By tameness at $p \mid N$ and the bound of Definition 1.1(iii), the root discriminant of $L / \mathbb{Q}$ satisfies $\varrho_{L}<\ell^{1+\frac{1}{\ell-1}} N^{1-\frac{1}{\ell}}$. More precisely,

$$
\operatorname{ord}_{p}\left(\varrho_{L}\right) \leq 1-\ell^{-1} \text { for all } p \mid N \text { and } \operatorname{ord}_{\ell}\left(\varrho_{L}\right)<1+(\ell-1)^{-1} \text {. }
$$

Proposition 6.2. For $\ell \leq 13$, the maximal $(\ell, 1)$-controlled extension $L$ is $\mathbb{Q}\left(\mu_{2 \ell}\right)$. Under GRH, the same is true for $\ell=17$ and 19 .

Proof. For $\ell$ odd, $\mathbb{Q}\left(\boldsymbol{\mu}_{\ell}\right) \subseteq L$ and $n=[L: \mathbb{Q}]$ is a multiple of $\ell-1$. From (6.1) and [18, we find $M$ in Table 1 below such that $n \leq(\ell-1) M$. If $\ell=13,17,19$, we see that $M<\ell$, so $L / \mathbb{Q}$ is tame at $\ell$ and $\varrho_{L} \leq \ell^{1-\alpha}$, with $\alpha=((\ell-1) M)^{-1}$. One gets a new bound $n \leq(\ell-1) M^{\prime}$ with $M^{\prime} \leq 5$. If $\ell \leq 11$, we have $M \leq 5$. In both cases, $L$ is abelian over $\mathbb{Q}\left(\boldsymbol{\mu}_{2 \ell}\right)$, and so $L=\mathbb{Q}\left(\boldsymbol{\mu}_{2 \ell}\right)$ by class field theory [3, Lem. 2.2]. Use $\mathbb{Q}(i) \subseteq L$ for $\ell=2$.

TABLE 1. Odlyzko bounds for $(\ell, 1)$-controlled fields

\begin{tabular}{|c||c|c|c|c|c|c|c|c|}
\hline$\ell$ & 2 & 3 & 5 & 7 & 11 & 13 & 17 & 19 \\
\hline$\varrho_{L} \leq$ & 4 & 5.197 & 7.477 & 9.682 & 13.981 & 16.099 & 20.294 & 22.377 \\
\hline$M$ & 2 & 3 & 3 & 3 & 5 & 7 & 8 & 10 \\
\hline
\end{tabular}

Now suppose $L$ is maximal $(2, N)$-controlled, so $\varrho_{L}<4 N^{\frac{1}{2}}$ by (6.1). If $n=[L: \mathbb{Q}]$ is finite, [18, Tables 3,4] provides $B, E$, depending on a parameter $b$, such that $\varrho_{L}>B e^{-\frac{E}{n}}$. In Table 2 below, we find a best bound for $n<E / \log \left(B / 4 N^{\frac{1}{2}}\right)$ by varying $B>4 N^{\frac{1}{2}}$, unconditionally for $N \leq 21$ and under GRH for larger $N$.

If $V$ is an irreducible semistable $\mathbb{F}_{2}\left[G_{\mathbb{Q}}\right]$-module good outside $S$ and $N_{S} \mid N$, then $\operatorname{Gal}(\mathbb{Q}(V) / \mathbb{Q})$ factors through $\bar{G}=G / H$, where $H$ is the maximal normal 
2-subgroup of $G=\operatorname{Gal}(L / \mathbb{Q})$. For odd $N \leq 79$ and $N=97$, we find a subfield $F$ of $L$ containing $L^{H}$ by composing a solvable extension of $\mathbb{Q}$ with a subfield of $\mathbb{Q}\left(J_{0}(N)[2]\right)$. Then we use the improvements in $\$ 5$ on the bound (6.1) for $\varrho_{L}$, together with the Odlyzko tables and Magma [2] to control $[L: F]$.

TABLE 2. Bounds on $n=[L: \mathbb{Q}]$ for $(2, N)$-controlled fields $L$

\begin{tabular}{|c||c|c|c|c|c|c|c|c|c|c|c|}
\hline$N$ & 3 & 5 & 7 & 11 & 13 & 15 & 17 & 19 & 21 & 23 & 29 \\
\hline$n \leq$ & 10 & 16 & 22 & 42 & 56 & 74 & 100 & 138 & 192 & 98 & 155 \\
\hline \hline$N$ & 31 & 33 & 35 & 37 & 39 & 41 & 43 & 47 & 51 & 53 & 55 \\
\hline$n \leq$ & 181 & 210 & 244 & 284 & 330 & 385 & 449 & 615 & 852 & 1007 & 1196 \\
\hline \hline$N$ & 57 & 59 & 61 & 65 & 67 & 69 & 71 & 73 & 77 & 79 & 97 \\
\hline$n \leq$ & 1427 & 1710 & 2061 & 3046 & 3743 & 4638 & 5800 & 7332 & 12042 & 15766 & 470652 \\
\hline
\end{tabular}

Theorem 6.3 (GRH). Let $V$ be semistable and irreducible over $\mathbb{F}_{2}$. If $V$ is bad exactly over $S$ and $N=N_{S} \leq 79$ or $N=97$, the following hold:

i) no such $V$ exists for $N$ in $\{3,5,7,13,15,17,21,33,39,41,55,57,65,77\}$;

ii) $V$ is unique and $\operatorname{dim} V=2$ for $N$ in $\{11,19,23,29,31,35,37,43,51,53,61\}$;

iii) $V$ is unique for $N$ in $\{23,31,47,71\}$;

iv) $V$ is an irreducible $\mathbb{F}_{2}[\bar{G}]$-module with $\bar{G}=D_{9}, D_{3} \times \mathcal{A}_{5}, \mathcal{A}_{5}, D_{3} \times D_{5}, \mathrm{SL}_{2}\left(\mathbb{F}_{8}\right)$ when $N=59,67,73,79,97$ respectively.

Remark 6.4. Aside from $\mathbb{F}_{2}$, there are exactly two irreducible $\mathbb{F}_{2}\left[\mathcal{A}_{5}\right]$-modules, both 4-dimensional, occurring as a submodule $V_{1}$ and quotient module $V_{2}$ of the permutation module. The non-trivial $\mathbb{F}_{2}\left[\mathrm{SL}_{2}\left(\mathbb{F}_{8}\right)\right]$-modules have dimensions 6,8 and 12 . Further, the irreducible modules for $G_{1} \times G_{2}$ are the tensor products of irreducibles for $G_{1}$ and $G_{2}$.

Sketch of the proof. In (i), $G$ is a 2 -group, except for $33,55,57,77$, when $\bar{G} \simeq D_{3}$ has a representation whose conductor, 11 or 19 , divides $N$ properly. In (ii), $V \simeq$ $C_{N}$ [2] for an elliptic curve $C_{N}$ of conductor $N$, except that $V \simeq J_{0}(29)[\sqrt{2}]$ for $N=29$. In (iii), $V$ is the $\mathbb{F}_{2}\left[D_{h}\right]$-module of dimension $h-1$ induced by the Hilbert class field over $\mathbb{Q}(\sqrt{-N})$ of class number $h=3,3,5,7$ corresponding to $N=23$, $31,47,71$ respectively.

$\mathbf{N}=59$ : The two irreducibles are the constituents of $J_{0}(59)$ [2], using an equation for $X_{0}(59)$, namely $y^{2}=f(x) g(x)$ with $f=x^{3}-x^{2}-x+2$ and

$$
g=x^{9}-7 x^{8}+16 x^{7}-21 x^{6}+12 x^{5}-x^{4}-9 x^{3}+6 x^{2}-4 x-4 .
$$

The Galois group of $g$ is $D_{9}$, and a root of $f$ gives a cubic subfield.

$\mathbf{N}=67$ : Let $V_{1}=C_{67}[2]$ and $V_{2}=J_{0}^{+}(67)[2]$. Then $\operatorname{Gal}\left(\mathbb{Q}\left(V_{2}\right) / \mathbb{Q}\right)=\mathrm{SL}_{2}\left(\mathbb{F}_{4}\right)$ and $\left[L: \mathbb{Q}\left(V_{1}, V_{2}, i\right)\right] \leq 2$.

We provide more details for $N=73,77,79$ and 97 . Let $E$ be the maximal abelian extension of $\mathbb{Q}$ in $L$. Since $G$ is generated by involutions, $E / \mathbb{Q}$ is the elementary 2-extension generated by $i$ and $\sqrt{p}$ as $p$ ranges over $S$.

Lemma 6.5. Let $M \supset F$ be subfields of $L$ containing $E$ and Galois over $\mathbb{Q}$. Set $T=\operatorname{Gal}(M / F)$ and assume $\lambda \mid 2$ is totally ramified of odd degree $t=|T|>1$ in $M / F$. Then $t=3$ and the residue degree $f_{\lambda}(E / \mathbb{Q})=2$. 
Proof. Since the image of $\alpha: \operatorname{Gal}(M / \mathbb{Q}) \rightarrow \operatorname{Aut}(T)$ by conjugation is abelian, $E$ contains $M_{0}=L^{\text {ker } \alpha}$, and so $f=f_{\lambda}\left(M_{0} / \mathbb{Q}\right) \leq f_{\lambda}(E / \mathbb{Q}) \leq 2$. Any Frobenius in $\mathcal{D}_{\lambda}\left(M / M_{0}\right)$ acts trivially on $T$. Thus $2^{f} \equiv 1(\bmod t)$ and the claim ensues.

Remark 6.6. Let $M \supseteq F$ be subfields of $L$ containing $\mathbb{Q}(i, \sqrt{N})$ and Galois over $\mathbb{Q}$. Denote the residue, ramification and tame degree of $\lambda$ in $F / \mathbb{Q}$ by $f_{0}, e_{0}$ and $t_{0}$ respectively. Given an a priori bound $[M: F] \leq b$, consider possible factorizations $[M: F]=2^{s} t_{1} u_{1}$, where $2^{s}$ is the degree of wild ramification, $t_{1}$ the degree of tame ramification and $u_{1}=f_{1} g_{1}$ the unramifed (inert and split) degree of $\lambda$ in $M / F$. The resulting tame ramification in $M / \mathbb{Q}$ requires that the completion $M_{\lambda}$ contain $\boldsymbol{\mu}_{t_{0} t_{1}}$, and so $2^{f_{0} f_{1}} \equiv 1\left(\bmod t_{0} t_{1}\right)$.

For each $s$ with $0 \leq s \leq \log _{2} b$, let $t_{1} \geq 1$ run through odd integers at most $b / 2^{s}$. Set $\beta=\left(c_{F} t_{1}+1\right) /\left(2^{s} t_{1} e_{0}\right)$, as in Remark [5.9, and let $n_{\beta}$ be the Odlyzko bound on $[M: \mathbb{Q}]$ when $\varrho_{M} \leq 2^{2-\beta} \sqrt{N}$. Then $1 \leq g_{1} \leq n_{\beta} /\left(2^{s} t_{1} f_{1}[F: \mathbb{Q}]\right)$. Values of $s, t_{1}, f_{1}$ not satisfying the congruence and inequality above are ruled out.

Let $E_{1}$ be the maximal subfield of $L$ abelian over $E$. By Lemmas 5.6(ii) and 5.8, the ray class conductor of $E_{1} / E$ divides $(1+i)^{2} \mathcal{O}_{E}$. Then class field theory or Magma gives Table 3 below.

TABle 3. Decomposition type of $\lambda \mid 2$ in $E_{1}$

\begin{tabular}{|c|c|c|c|c|}
\hline$N$ & $\mathrm{Gal}\left(E_{1} / E\right)$ & $e_{\lambda}\left(E_{1} / \mathbb{Q}\right)$ & $f_{\lambda}\left(E_{1} / \mathbb{Q}\right)$ & $g_{\lambda}\left(E_{1} / \mathbb{Q}\right)$ \\
\hline 73 & $C_{4}$ & 4 & 2 & 2 \\
\hline 77 & $C_{6}$ & 6 & 2 & 4 \\
\hline 79 & $C_{15}$ & 2 & 5 & 6 \\
\hline 97 & $C_{4}$ & 4 & 2 & 2 \\
\hline
\end{tabular}

$\mathbf{N}=73$ : The Jacobian $J_{0}^{+}(73)$ has RM by $\mathbb{Q}(\sqrt{5})$ and the Galois group of its 2-division field $K$ is $\mathrm{SL}_{2}\left(\mathbb{F}_{4}\right) \simeq \mathcal{A}_{5}$. For the 5 primes over 2 in $K, f_{\lambda}(K / \mathbb{Q})=3$ and Frobenius acts irreducibly on $\mathcal{I}_{\lambda}(K / \mathbb{Q}) \simeq C_{2}^{2}$. Since Frobenius is reducible on $\mathcal{I}_{\lambda}\left(E_{1} / \mathbb{Q}\right) \simeq C_{2}^{2}$, we have $\mathcal{I}_{\lambda}(F / \mathbb{Q}) \simeq C_{2}^{4}$ for the compositum $F=E_{1} K$, thus $[F: \mathbb{Q}]=960$. By Table $2,[L: \mathbb{Q}]=960 r \leq 7332$, so $r \leq 7$. Lemma 6.5 implies the tame degree $t_{\lambda}(L / F)=1$, so $e_{\lambda}(L / F)$ divides 4 . Finally, $[L: F]$ divides 4 by Remark 6.6.

$\mathbf{N}=77$ : In the $\mathcal{S}_{3}$-field $K_{0}=\mathbb{Q}\left(J_{0}(11)[2]\right)=\mathbb{Q}(\sqrt{-11}, \theta)$, with $\theta^{3}-2 \theta^{2}+2=0$, the decomposition type over 2 is $e_{\lambda}=3, f_{\lambda}=2, g_{\lambda}=1$. If $K=E(\theta)=K_{0}(i, \sqrt{-7})$, then $\operatorname{Gal}(K / \mathbb{Q}) \simeq C_{2} \times C_{2} \times \mathcal{S}_{3}$ and $\mathcal{I}_{\lambda}(K / \mathbb{Q}) \simeq C_{6}$, so $m_{K}=3$ by Lemma 5.6 (i). If $F$ is the maximal subfield of $L$ abelian over $K$, the ray class conductor of $F / K$ divides $(1+i)^{4} \mathcal{O}_{K}=4 \mathcal{O}_{K}$ by Lemma 5.8. Then $\operatorname{Gal}(F / K) \simeq C_{2} \times C_{2} \times C_{4}$ and the decomposition type of 2 is $e_{\lambda}(F / \mathbb{Q})=48, f_{\lambda}(F / \mathbb{Q})=2$ and $g_{\lambda}(F / \mathbb{Q})=4$.

A group of order $3 \cdot 2^{a}$ admits a unique quotient isomorphic to $C_{3}$ or $\mathcal{S}_{3}$. If $\left[L: K_{0}\right]=3 \cdot 2^{a}$, there is a $C_{3}$ or $\mathcal{S}_{3}$ extension of $K_{0}$. The latter provides a central quadratic $M_{0} / K_{0}$, with $M_{0} / \mathbb{Q}$ Galois and $\operatorname{Gal}\left(M_{0} / \mathbb{Q}\right) \simeq D_{6}$. In both cases, we find that $\operatorname{Gal}\left(M_{0} K / K\right) \simeq C_{3}$, contradicting $[F: K]=16$.

We claim that $\operatorname{Gal}(L / F)$ is a 2-group. If not, since $[L: F] \leq 31$ from Table 2 and $[L: F] \neq 3 \cdot 2^{a}$, the wild ramification degree $\left|\mathcal{I}_{\lambda}(L / F)_{1}\right|$ divides 4 . Example 5.7 and (5.4) imply that $\mathcal{I}_{\lambda}(F / \mathbb{Q})_{9} \neq 1$. Use Remark 6.6 with $c_{F} \geq 9$ to show that the only remaining case is $[L: F]=10$, with tame degree $t_{\lambda}(L / F)=5$ and wild degree 2. It is precluded by Lemma 6.5. 
Thus the kernel of the surjection $G \stackrel{\eta}{\rightarrow} \operatorname{Gal}\left(\mathbb{Q}\left(J_{0}(11)[2]\right) / \mathbb{Q}\right) \simeq \mathcal{S}_{3}$ is a 2-group and irreducible representations $V$ of $G$ factor through Image $\eta$, of conductor 11, so there is no $V$ of conductor 77 .

$\mathbf{N}=79$ : The strict class fields $H^{ \pm}$of $\mathbb{Q}(\sqrt{ \pm 79})$ have respective orders 3 and 5 , and so $E_{1}=H^{+} H^{-}$. Let $K^{ \pm}$be the maximal subfields of $L$ abelian respectively over $H^{ \pm}(i)$. Since $e_{\lambda}\left(H^{ \pm}(i) / \mathbb{Q}\right)=2$, the ray class conductors of $K^{ \pm} / H^{ \pm}(i)$ divide $(1+i)^{2} \mathcal{O}_{H^{ \pm}(i)}$ by Lemma 5.8. Magma provides the following information:

$$
\begin{aligned}
& \operatorname{Gal}\left(K^{+} / H^{+}(i)\right) \simeq C_{2}^{2} \times C_{3}, \quad \text { with } e_{\lambda}=2, f_{\lambda}=2, g_{\lambda}=3 ; \\
& \operatorname{Gal}\left(K^{-} / H^{-}(i)\right) \simeq C_{2}^{4} \times C_{5}, \quad \text { with } e_{\lambda}=16, f_{\lambda}=5, g_{\lambda}=1
\end{aligned}
$$

If $\operatorname{Gal}\left(E_{1} / E\right)=\langle\tau\rangle$, then $\tau^{5}$ and $\tau^{3}$ act trivially on $K^{+}$and $K^{-}$respectively. Hence $\tau$ is trivial on $K^{+} \cap K^{-}$and $\left(K^{+} \cap K^{-}\right) / E$ is abelian. Since $K^{+} \cap K^{-}$ contains $E_{1}$, equality holds by maximality of $E_{1}$. For $F=K^{+} K^{-}$, we therefore have $\left[F: E_{1}\right]=2^{6},[F: \mathbb{Q}]=3840$ and $[L: \mathbb{Q}]=3840 r$, with $r \leq 3$. Because Frobenius acts irreducibly on $\mathcal{I}_{\lambda}\left(K^{-} / E_{1}\right) \simeq C_{2}^{4}$ but trivially on $\mathcal{I}_{\lambda}\left(K^{+} / E_{1}\right) \simeq C_{2}$, we see that $\mathcal{I}_{\lambda}\left(F / E_{1}\right) \simeq C_{2}^{5}$ and $e_{\lambda}(F / \mathbb{Q})=64$. By Lemma 6.5, $t_{\lambda}(L / F)=1$, so $[L: F] \leq 2$ by Remark 6.6. Thus the kernel of $G \rightarrow \operatorname{Gal}\left(H^{+} / \mathbb{Q}\right) \times \operatorname{Gal}\left(H^{-} / \mathbb{Q}\right) \simeq D_{3} \times D_{5}$ is a 2-group.

$\mathbf{N}=97:$ There is a subfield $K$ of $\mathbb{Q}\left(J_{0}(97)[2]\right)$ with $\operatorname{Gal}(K / \mathbb{Q}) \simeq \mathrm{SL}_{2}\left(\mathbb{F}_{8}\right)$ and decomposition type $e_{\lambda}(K / \mathbb{Q})=8, f_{\lambda}(K / \mathbb{Q})=7, g_{\lambda}(K / \mathbb{Q})=9$. A Frobenius in $\mathcal{D}_{\lambda}(K / \mathbb{Q})$ acts irreducibly on $\mathcal{I}_{\lambda}(K / \mathbb{Q}) \simeq C_{2}^{3}$ but reducibly on $\mathcal{I}_{\lambda}\left(E_{1} / \mathbb{Q}\right) \simeq C_{2}^{2}$, so $\mathcal{I}_{\lambda}(F / \mathbb{Q}) \simeq C_{2}^{5}$ for the compositum $F=E_{1} K$. Table 2 implies that $[L: F] \leq 58$, since $[F: \mathbb{Q}]=504 \cdot 16=8064$. Thus the dimensions of irreducible representations of $\mathrm{SL}_{2}\left(\mathbb{F}_{8}\right)$ over $\mathbb{F}_{p}$ for small $p$ force the action of $\mathrm{Gal}\left(F / E_{1}\right)$ on the maximal abelian quotient of $\operatorname{Gal}(L / F)$ to be trivial. But no central extension of $\mathrm{SL}_{2}\left(\mathbb{F}_{8}\right)$ is perfect [1, 11. Hence $L$ is the compositum of $F$ with a solvable extension of $E_{1}$. The ray class extension of $E_{1}$ whose conductor divides $\prod \lambda^{2}$, as $\lambda$ runs over the primes above 2 in $\mathcal{O}_{E_{1}}$, turns out to be trivial, whence $L=F$ by Lemmas 5.6 (ii) and 5.8.

The asymptotic root discriminant bound of [19] is $8 \pi e^{\gamma} \approx 44.763$, where $\gamma$ is Euler's constant. Hence, by (6.1), the degree of $L$ is finite for odd squarefree $N$ at most 123. It would thus be entertaining to find $L$ when $N=127$. Below, we exhibit a subfield $F$ of $L$ of degree 161280 whose root discriminant $\varrho_{F}$ just exceeds the asymptotic bound.

To construct $F$, we begin the solvable tower with $E_{0}=E=\mathbb{Q}(i, \sqrt{127})$ and find successive maximal abelian extensions $E_{j+1} / E_{j}$ in $L / \mathbb{Q}$. For ray class conductor $(1+i)^{2} \mathcal{O}_{E}$, we have $\left[E_{1}: E\right]=5$. Thus $E_{1}$ is the compositum of $\mathbb{Q}(i)$ and the Hilbert class field over $\mathbb{Q}(\sqrt{-127})$. Now $e_{\lambda}\left(E_{1} / \mathbb{Q}\right)=2$, so the ray class conductor of $E_{2} / E_{1}$ divides $(1+i)^{2} \mathcal{O}_{E_{1}}$ and we have $\operatorname{Gal}\left(E_{2} / E_{1}\right)=C_{2}^{4}$. Moreover, any Frobenius in $\mathcal{D}_{\lambda}\left(E_{2} / \mathbb{Q}\right)$ has irreducible action of order 5 on this ray class group. The decomposition type over 2 is $e_{\lambda}\left(E_{2} / \mathbb{Q}\right)=32, f_{\lambda}\left(E_{2} / \mathbb{Q}\right)=5, g_{\lambda}\left(E_{2} / \mathbb{Q}\right)=2$. The ray class conductor of $E_{3} / E_{2}$ divides $\prod \lambda^{2}$, as $\lambda$ runs over the primes of $\mathcal{O}_{E_{2}}$ above 2 , but we do not know whether $E_{3}=E_{2}$.

Also, there is a subfield $K$ of $\mathbb{Q}\left(J_{0}(127)[2]\right)$ with $\operatorname{Gal}(K / \mathbb{Q}) \simeq \mathrm{SL}_{2}\left(\mathbb{F}_{8}\right)$ and $e_{\lambda}(K / \mathbb{Q})=8, f_{\lambda}(K / \mathbb{Q})=7, g_{\lambda}(K / \mathbb{Q})=9$. Any Frobenius in $\mathcal{D}_{\lambda}(K / \mathbb{Q})$ has irreducible action of order 7 on $\mathcal{I}_{\lambda}(K / \mathbb{Q}) \simeq C_{2}^{3}$. For the compositum $F=E_{2} K$, of degree $320 \cdot 504=161280$, we therefore have $\mathcal{I}_{\lambda}(F / \mathbb{Q}) \simeq C_{2}^{8}$, and so $c_{F}=m_{F}=1$ by Lemma 5.6. Then $\varrho_{F}=2^{2-\frac{1}{128}} \sqrt{127} \approx 44.834$ by Remark [5.9. 


\section{REFERENCES}

[1] M. Aschbacher, Finite Group Theory, Cambridge University Press, 1986. MR895134 (89b:20001)

[2] W. Bosma, J. Cannon and C. Playoust. The Magma algebra system. I. The user language. J. Symb. Comp., 24 (1997) 235-265. MR 1484478

[3] A. Brumer and K. Kramer, Semistable Abelian varieties with small division fields. Galois Theory and Modular Forms, Hashimoto, Miyake, Nakamura, eds., Kluwer, 2003, 13-38. MR2059756(2005m:11106)

[4] A. Brumer and K. Kramer, Paramodular abelian varieties of odd conductor, math arXiv:1004.4699.

[5] F. Diaz y Diaz, Tables minorant la racine $n$-ième du discriminant d'un corps de degré $n$, Ph.D. Thesis, Publ. Math. Orsay, 1980.

[6] R.H. Dye, Interrelations of symplectic and orthogonal groups in characteristic two, J. of Algebra, 59 (1979) 202-221. MR541675 (81c:20028)

[7] J.-M. Fontaine, Il n'y a pas de variété abélienne sur $\mathbb{Z}$, Invent. Math., 81 (1985) 515-538. MR.807070 (87g:11073)

[8] B. H. Gross and J. Harris, On some geometric constructions related to theta characteristics. Contributions to automorphic forms, geometry, and number theory, Hida et al., eds., Johns Hopkins, 2004, 279-312. MR2058611 (2005h:14079)

[9] A. Grothendieck, Modèles de Néron et monodromie. Sém. de Géom. 7, Exposé IX, Lecture Notes in Math., 288, Springer-Verlag, 1973.

[10] F. Hajir and C. Maire, Extensions of number fields with wild ramification of bounded depth, Inter. Math. Res. Notices, 13 (2002) 667-696. MR1890847 (2002m:11096)

[11] B. Huppert, Endliche Gruppen. I, Springer-Verlag, 1967. MR0224703 (37:302)

[12] J. Jones, Wild ramification bounds and simple group Galois extensions ramified only at 2, Proc. Amer. Math. Soc., 139 (2011) 807-821. MR2745634

[13] J. Jones and D. Roberts, Local fields, J. Symbolic Computation, 41(1) (2006) 80-97. MR2194887(2006k:11230)

[14] J. Jones and D. Roberts, Galois number fields with small root discriminant, J. Number Theory, 122 (2007) 379-407. MR2292261(2008e:11140)

[15] W.M. Kantor, Subgroups of classical groups generated by long roots, Trans. Amer. Math. Soc., 248 (1979) 347-379. MR522265 (80g:20057)

[16] G. Kemper and G. Malle, The finite irreducible linear groups with polynomial ring of invariants, Transformation Groups, 2 (1997) 57-89. MR.1439246 (98a:13012)

[17] J. McLaughlin, Some subgroups of $\operatorname{SL}_{n}\left(\mathbb{F}_{2}\right)$, Ill. J. Math., 13 (1969) 108-115. MR.0237660 (38:5941)

[18] A. Odlyzko, Lower bounds for discriminants of number fields. II, Tôhoku Math. J., 29 (1977) 209-216. MR0441918 (56:309)

[19] A. Odlyzko, Bounds for discriminants and related estimates for class numbers, regulators and zeros of zeta functions: A survey of recent results, Sém. Théorie Nombres Bordeaux, (2)2(1) (1990) 119-141. MR1061762 (91i:11154)

[20] R. Schoof, Abelian varieties over cyclotomic fields with everywhere good reduction, Math. Ann., 325 (2003) 413-448. MR.1968602 (2005b:11076)

[21] J.-P. Serre, Local Fields, Graduate Texts in Math., 67, Springer-Verlag, 1979. MR0554237 (82e:12016)

[22] A. E. Zalesskii and V. N. Serežkin, Finite linear groups generated by reflections, Math. USSR Izv., 17 (1981) 477-503. MR603578 (82i:20060)

Department of Mathematics, Fordham University, Bronx, New York 10458

E-mail address: brumer@fordham.edu

Department of Mathematics, Queens College and the Graduate Center (CUNY), 65-30 Kissena Boulevard, Flushing, New York 11367

E-mail address: kkramer@gc.cuny.edu 